\title{
Insecticide resistance of Dengue vectors in South East Asia: a systematic review
}

\author{
Mohd Rohaizat Hassan, Noor Atika Azit, Suhaiza Mohd Fadzil, Siti Rasidah Abd Ghani, \\ Norfazilah Ahmad, Azmawati Mohammed Nawi
}

Department of Community Health, Faculty of Medicine, Universiti Kebangsaan Malaysia, Jalan Yaacob Latiff, 56000 Cheras, Kuala Lumpur, Malaysia.

\section{Emails:}

Mohd Rohaizat Hassan: rohaizat@ppukm.ukm.edu.my; Noor Atika Azit: atika3008@gmail.com; Suhaiza Mohd Fadzil: suhaiza6378@gmail.com; Siti Rasidah Abd Ghani: sitirasidah3006@gmail.com; Norfazilah Ahmad: norfazilah@ppukm.ukm.edu.my

\begin{abstract}
Background: The insecticides used widely has led to resistance in the vector and impose a challenge to vector control operation.

Objectives: This review aims to analyse the distribution of insecticide resistance of dengue vectors in South East Asia and to describe the mechanism of insecticide resistance.

Methods: Literature search for articles published on 2015 to 2019 from PubMed, Scopus and ProQuest was performed. Total of 37 studies included in the final review from the initial 420 studies.

Results: Pyrethroid resistance was concentrated on the west coast of Peninsular Malaysia and Northern Thailand and scattered at Java Island, Indonesia while organophosphate resistance was seen across the Java Island (Indonesia), West Sumatera and North Peninsular Malaysia. Organochlorine resistance was seen in Sabah, Malaysia and scattered distribution in Nusa Tenggara, Indonesia. V1016G, S989P, F1269C gene mutation in Aedes Aegypti were associated with Pyrethroid resistance in Singapore and Indonesia. In Malaysia, over-expressed with monooxygenase P450 genes (CYP9J27, CYP6CB1, CYP9J26 and CYP9M4) Glutathione S-transferases, carboxylesterases commonly associated with pyrethroids resistance in Aedes Aegypti and CYP612 overexpressed in Aedes Albopictus. The genetic mutation in A302S in Aedes Albopictus was associated with organochlorine resistance in Malaysia.

Conclusions: Rotation of insecticide, integration with synergist and routine assessment of resistance profile are recommended strategies in insecticide resistance management.

Keywords: Insecticide resistance; vector management; Aedes; pyrethroid; mortality.

DOI: https://dx.doi.org/10.4314/ahs.v21i3.21

Cite as: Hassan MR, Azit NA, Fadzil SM, Abd Ghani SR, Ahmad N, Nawi AM. Insecticide resistance of Dengue vectors in South East Asia: a systematic review. Afri Health Sci. 2021;21(3). 1124-1140. bttps:// dx.doi.org/10.4314/abs.v21i3.21
\end{abstract}

\section{Introduction}

Aedes mosquito is an essential vector for many vector-borne infections. Aedes mosquito species mainly Aedes aegypti is the primary vector, and Aedes albopictus is the vector responsible for transmitting the dengue virus between people. There is four known dengue virus

\section{Corresponding author:}

Azmawati Mohammed Nawi,

Department of Community Health, Faculty of

Medicine, National University of Malaysia, Jalan

Yaacob Latiff,

56000 Cheras, Kuala Lumpur

Phone no: +60391458780

Email: azmawati@ppukm.ukm.edu.my serotype circulating in Asia, Africa and America designated as DENV-1, DENV-2, DENV-3 and DENV-4 transmitted by the Aedes mosquito ${ }^{1}$. Infection to one serotype will produce lifelong immunity to that serotype virus and cross-protection to other serotypes for a few months ${ }^{1}$. Secondary infection with other serotypes will lead to severe infection ${ }^{1}$.

Aedes mosquito species found between latitude $45^{\circ}$ North and $35^{\circ}$ South in tropics and subtropical countries which closely related to human habitat in urban area ${ }^{2}$. It breeds mostly in a man-made container such as used tyres, plastic container, tin container, and even a small cup can attract the female Aedes mosquito to breed. Aedes mosquito takes about 8 to 10 days to complete the life cycle from eggs to adult ${ }^{3}$. Female adult work is properly cited. 
Aedes mosquito has a strong affinity for human blood and prefers to bite human during dusk and dawn period. Due to its limited flying capacity, it is found mostly inside or outside home or buildings close to human ${ }^{3}$.

In 1998 Aedes mosquito was found throughout all continents including North America and Europe, and as the result of expanding geographical distribution, half of the world's population are at risk of dengue infection ${ }^{4}$. Before the 1970s only nine countries reporting severe dengue epidemics, however since the last five decades dengue fever is endemic in more than 125 countries with the western Pacific region, South-East Asia and America are the most affected countries ${ }^{5}$. It estimated that about 390 million dengue infection occur every year throughout the world with 20000 dengue-related death that is resulting in dengue considered as worsening public health problem ${ }^{5}$. Most countries in South East Asia has a high burden of dengue infection with 3 to 5 years cyclical episodes of epidemics ${ }^{6}$. In 2010, 354009 cases from WHO Western Pacific Region of South East Asian countries which include Cambodia, Lao, Malaysia, Singapore, Philippines and Vietnam with 1075 deaths were reported to World Health Organization $(\mathrm{WHO})^{6,7}$. Together with other South-East Asian countries, it contributes to $75 \%$ of disease burden 6 .

Environmental change, urbanisation, increase population density, travel and trade activities that allowing movement of the vectors and virus via modern transportation, the emergence of these viruses from their sylvatic reservoirs, has been recognised to contribute to the emergence and re-emergence of dengue in South Asia ${ }^{8}$. Aedes mosquito has a high vectorial capacity and undergone adaptive changes to achieve longevity and to survive in cooler climates ${ }^{2}$. Unplanned urbanisation and weak environmental management cause increase in potential breeding site for Aedes mosquito. High-density population in the capital city and travel activity further increased the dengue epidemic in South Asian countries.

Till date, there is no specific treatment for dengue infection and Dengvaxia vaccine is not widely used ${ }^{9}$. Source reduction, clean-up campaign and larviciding have been promoted widely; however, it was not very successful. Prevention and control of Aedes nowadays are based mainly on integrated vector management which includes integrated use of insecticide by space spraying to reduce the adult mosquito density, larviciding and environmental management ${ }^{10}$. Space-spraying using aerial and truck-mounted ultra-low volume (ULV) is now not recommended because of the low impact on mosquito density reduction and less cost-effective to implement it as a routine intervention ${ }^{11}$. There are four significant insecticides commonly used, namely pyrethroid, organophosphate, carbamate and organochlorine. Long term and extensively used of these insecticides in dengue control has led to the development of resistance in the vector, and this will impose a challenge to vector control operation?. Resistance defined as the ability in a strain of some organism to tolerate doses of a toxicant that would prove lethal to majority of individuals in a normal population of the same species owing to physiological or behavioural adaptation ${ }^{12}$. Insecticides resistance will often be led to the more frequent use of insecticide, higher dose and can harm the environment. Worldwide, insecticide resistance has been reported. For Aedes Aegypti, there is consistently high deltamethrin resistance in Brazil and French Guiana while scattered deltamethrin resistance of Aedes Aegypti was found not only in South East Asia, but also in the Middle Eastern region (Saudi Arabia) 9,13. Temephos resistance has been reported in Brazil, French Guiana and the Caribbean whereas lower resistance in some parts of Africa? While resistance to all four groups of insecticide for $A e$ des Albopictus in South East Asia'. Particular mechanism of insecticides resistance of the vector has pointed out. This includes reduced penetration of insecticide into the vector, metabolic resistance where there is enhanced enzymatic activity for biodegradation and sequestration of insecticides, a non-synonymous mutation affecting the proteins targeted by insecticides ${ }^{14,15}$ and behavioural resistance $^{12}$.

There is no recent published report on insecticide resistance of dengue vector that covers multiple types of insecticide specific to South East Asia. Some studies only report on pyrethroid resistance ${ }^{16}$, and others only study on resistant to insecticide at either larva or adult stage. More studies have done focussing on insecticides resistance for malaria vector compared to Aedes. Understanding of the current distribution of insecticide resistance, mechanism of resistance and alternative method to overcome the resistance will allow the implementer of vector control to make an appropriate decision on the appropriate choice of insecticide for the dengue control program. The findings also provide input for conducting surveillance and monitoring of insecticide resistance as well as to evaluate the effectiveness of vector management. Therefore, this systematic review will provide an analysis of the distribution of 
insecticide resistance of Aedes mosquito in South East Asia, to describe the mechanism of insecticide resistance of Aedes mosquito in South Asia as well as to identify the possible insecticidresistance management plan.

\section{Material \& methods}

\section{Search strategy and study selection}

A comprehensive search of literature from PubMed, Scopus and ProQuest was performed on the $1^{\text {st }}$ of April 2019 to search for relevant studies. PRISMA checklist used for the workflow of publications search. The text keywords used are "South East Asia" OR Malaysia OR Thailand OR Singapore OR Brunei OR Myanmar OR Cambodia OR Laos OR Philippines OR Indonesia OR "East Timo" OR Vietnam AND Aedes OR dengue OR aegypti OR albopictus AND pesticides OR insecticide OR "dengue control" OR fogging OR organophosphate OR pyrethroid OR larvicide OR temephos OR diphenylmethane AND resistance OR knockdown OR kdr OR mutation with year limit from 2014 to 2019. Articles retrieved from the database and compiled using Mendeley Desktop version 1.19.4 duplicates with $100 \%$ matched were removed by the software automatically. Next, the title of each article read by all authors and agreement obtained to exclude articles that did not match with the keywords. If there was any doubt, the abstract was retrieved and read to justify the decision. The abstracts of the articles distributed among the authors for assessment of inclusion and exclusion criteria. Then, selected full articles were retrieved and distributed to the authors. Two independent authors were responsible for examining and extracting the data for each article. At the disagreement point, the third author was consulted.

PubMed, Scopus and ProQuest searches identified 420 articles. Thirty-three duplicates were removed. By screening and reviewing for title and abstract, 55 potentially relevant articles were identified and retrieved for more detailed evaluation. Out of these 55 articles, 37 articles fulfilled all the inclusion and exclusion criteria, and subsequently, 18 articles excluded with reasons. There were three articles rejected because of reviewed articles, six articles not related to insecticide resistance, two articles are studies on human, two articles with no full text available, three not involve insecticides, and two articles did not examine on Aedes Aegypti or Aedes Albopictus species. The detail PRISMA flow diagram illustrated in Figure 1.
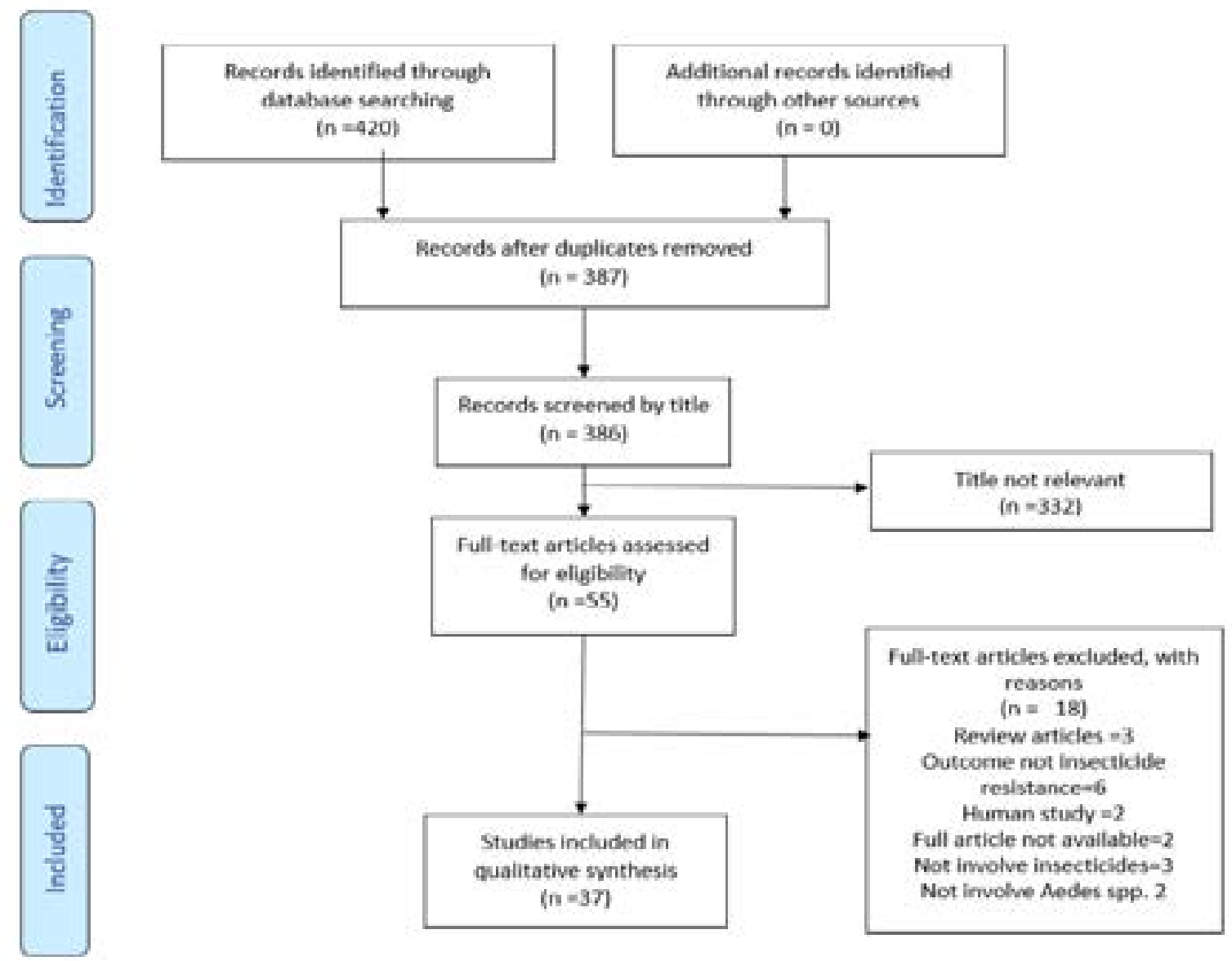

Figure 1: Process of Study Selection 


\section{Data Extraction and Data Synthesis}

Data extraction was done by the authors independently using a predetermined data collection form. The data will then have crossed checked by all authors to minimise errors. Resistance data obtained from all the included articles. In order to find the geographical location of the study sites, the latitude and longitude extracted from the reported article. If the data is not available, the latitude and the longitude of the study site generated using latlong.net (Latlong coordinates reference for World Geodetic System WGS84 standard), based on the study location's name specified in the articles. In this study, the insecticide resistance status categorised based on the latest WHO recommendation ${ }^{17}$, which are more than 98\% 24 hours mortality is categorised as "susceptible", less than 98\% 24 hours mortality suggest "resistance".

The spatial data analysed with QGis software version 3.6. The resistance status was mapped according to the class of insecticide, which are pyrethroid, organophosphate and organochlorine. The data were plotted against the latitude and the longitude to obtain the spatial distribution of insecticide resistance.

\section{Results}

\section{Descriptive Analysis}

Thirty-seven (37) studies selected in this review, pub- lished from the year 2015 to 2019. Thirteen (12) articles were from Malaysia, twelve (12) from Indonesia, six (6) from Thailand, one (1) from Laos, one (1) from Vietnam, three (3) from Singapore, one (1) from Cambodia, one (1) includes Asian countries.

The insecticides studied from pyrethroid, organophosphate, organochlorine and carbamate classes. The type of insecticides was varied from one study to another, and with different concentration. The main resistance measurement was a percentage of 24 hours mortality, Knocked Down Time for 50\% (KT50) or Knocked Down Time for 95\% (KT95), and specific gene mutation. In term of the year study conducted, the earliest year stated in the articles was in 2006. However, not all articles mentioned the year of the resistance studies were conducted. However, the authors estimated the studies should be on average less than years from the publication date ${ }^{18}$. There is the variability of the dosage for specific pesticides tested for the resistance across the study. Most of the studies using the baseline dosage stated by the World Health Organization (WHO) ${ }^{17}$; however, some studies examined the resistance with an escalated dosage of the pesticides. In term of Aedes population, adult or larvae or both were tested. Besides, the studies were specific to either Aedes Albopictus or Aedes Aegypti or both. Table 1 summarises the general information of the included studies.

Table 1: Summary of the Characteristics of Included Studies

\begin{tabular}{|c|c|}
\hline Subject & Description \\
\hline Countries & $\begin{array}{l}\text { Malaysia (12), Indonesia (12), Thailand } \\
\text { (6), Laos (1), Vietnam (1), Singapore (3), } \\
\text { Cambodia (1), Asian (1) }\end{array}$ \\
\hline Insecticide studied & $\begin{array}{l}\text { Pyrethroid, organophosphate, } \\
\text { organochlorine, carbamate }\end{array}$ \\
\hline $\begin{array}{l}\text { Resistance } \\
\text { measurement }\end{array}$ & $\begin{array}{c}\% \text { mortality (majority), } \mathrm{KT}_{50} \text { or } \mathrm{KT}_{95}, \\
\text { Gene mutation (16) }\end{array}$ \\
\hline $\begin{array}{l}\text { Ranges of the year } \\
\text { of study }\end{array}$ & Earliest 2006- 2017(estimated) \\
\hline $\begin{array}{l}\text { Dosage } \\
\text { comparison }\end{array}$ & $\begin{array}{l}\text { Various, majority studied baseline } \\
\text { dosage provided by who or self-determined } \\
\text { based on diagnostic dose calculation }\end{array}$ \\
\hline Aedes population & $\begin{array}{l}\text { Adult or larvae, or both, species- } \\
\text { specific }\end{array}$ \\
\hline
\end{tabular}

\section{Mapping the Distribution of Resistance Status}

Figure 2 illustrates the distribution of overall insecticide resistance irrespective of the class of the pesticides. Majority of the study used discriminating concentrations of the relevant insecticide from WHO bioassay ${ }^{16}{ }^{17,6}$, while some were self determinedself-determined based on diagnostic dose calculation. The distribution of the resistance status represented with the percentage of 24 
hours mortality of the mosquitoes. The $98 \%$ mortality indicates susceptibility, whereby $90 \%$ to $97 \%$ mortality means the resistance is suggested and need further confirmation. For below $90 \%$ mortality, resistance is indicated and did not need further confirmation test.

The insecticide resistance was distributed in a concentrated form at the Peninsular Malaysia and Sumatera Barat region. There is a mixed pattern (susceptibility and resistance) on Java Island and Northern Thailand. Meanwhile, some studies showed susceptibility towards insecticides in West Malaysia, Vietnam and Laos. However, this data was solely on the published data. This means that it displays the presence of the status but did not describe the overall situation of each locality.

The pyrethroid resistance illustrated in Figure 3 and concentrated on the west coast of Peninsular Malaysia, as well as Northern Thailand. None of the published data on resistance in Vietnam and only one location in Laos. There is scattered distribution throughout Java Island, Indonesia. While for organophosphate, resistance represented by clustered distribution in Sumatera Barat, Indonesia and Sabah, East of Malaysia (Figure 4). Similar pattern as pyrethroid resistance seen across Java Island (Indonesia), for Organophosphate Resistance. Besides, study sites at Sumatera Barat and Northern part of Peninsular Malaysia showed similar resistance to pyrethroid and organophosphate, which suggest the possibility of cross-resistance. Clustered distribution in Sabah, East of Malaysia. Scattered distribution in Nusa Tenggara Barat, Indonesia. However, there is no data on Organochlorine resistance published from Laos, Vietnam and Thailand (Figure 5). Table 2 is the summary of the included studies in this review.

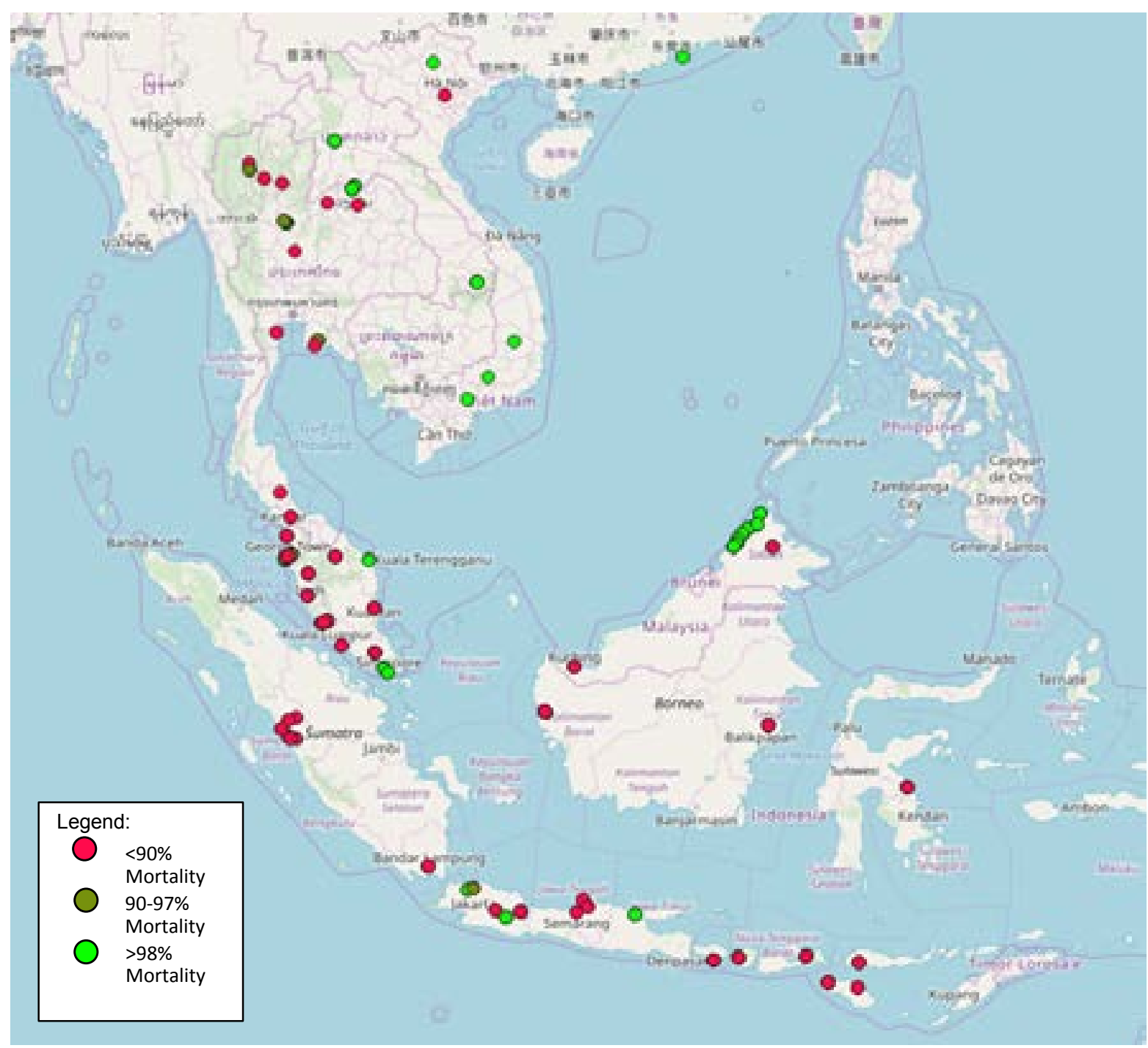

Figure 2 The Distribution of 24 Hours Mortality of Insecticide Resitance, Irrespective of the Class of the Pesticide 


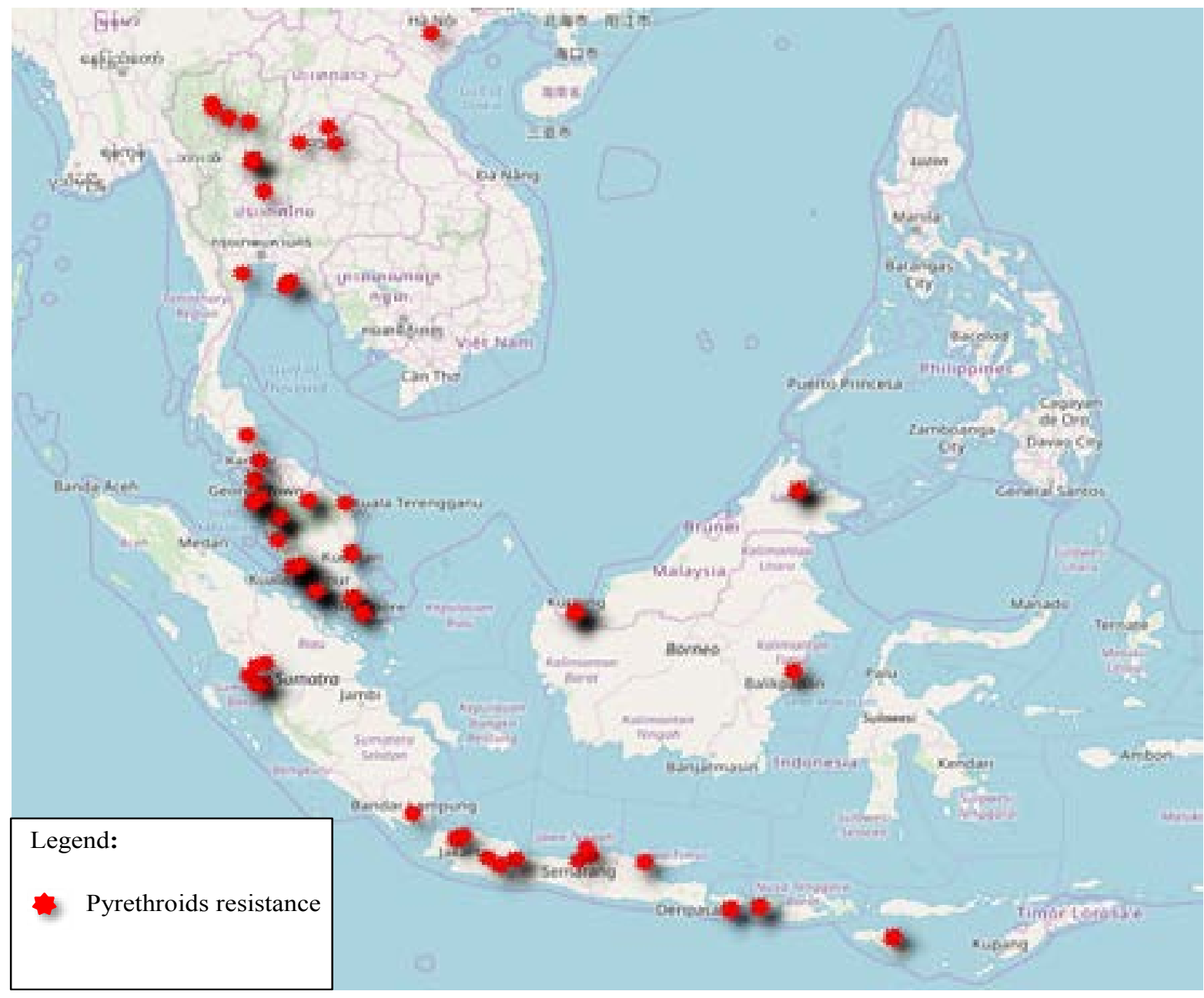

Figure 3 The Distribution of Pyrethroids Resistance Published from 2015 to 2019

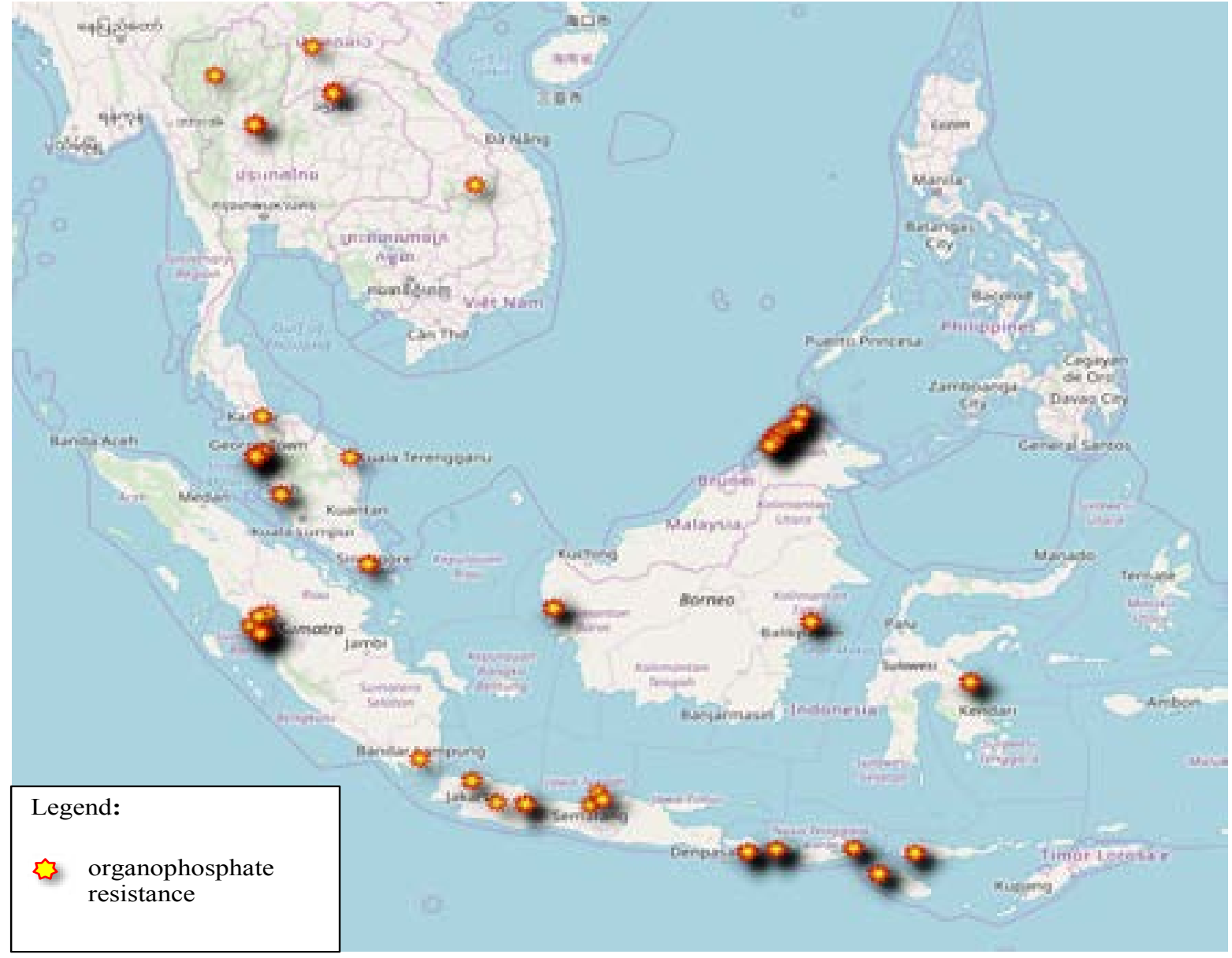

Figure 4 Organophosphate Resistance Based on Published Data from 2015 to 2019

African Health Sciences, Vol 21 Issue 3, September, 2021 


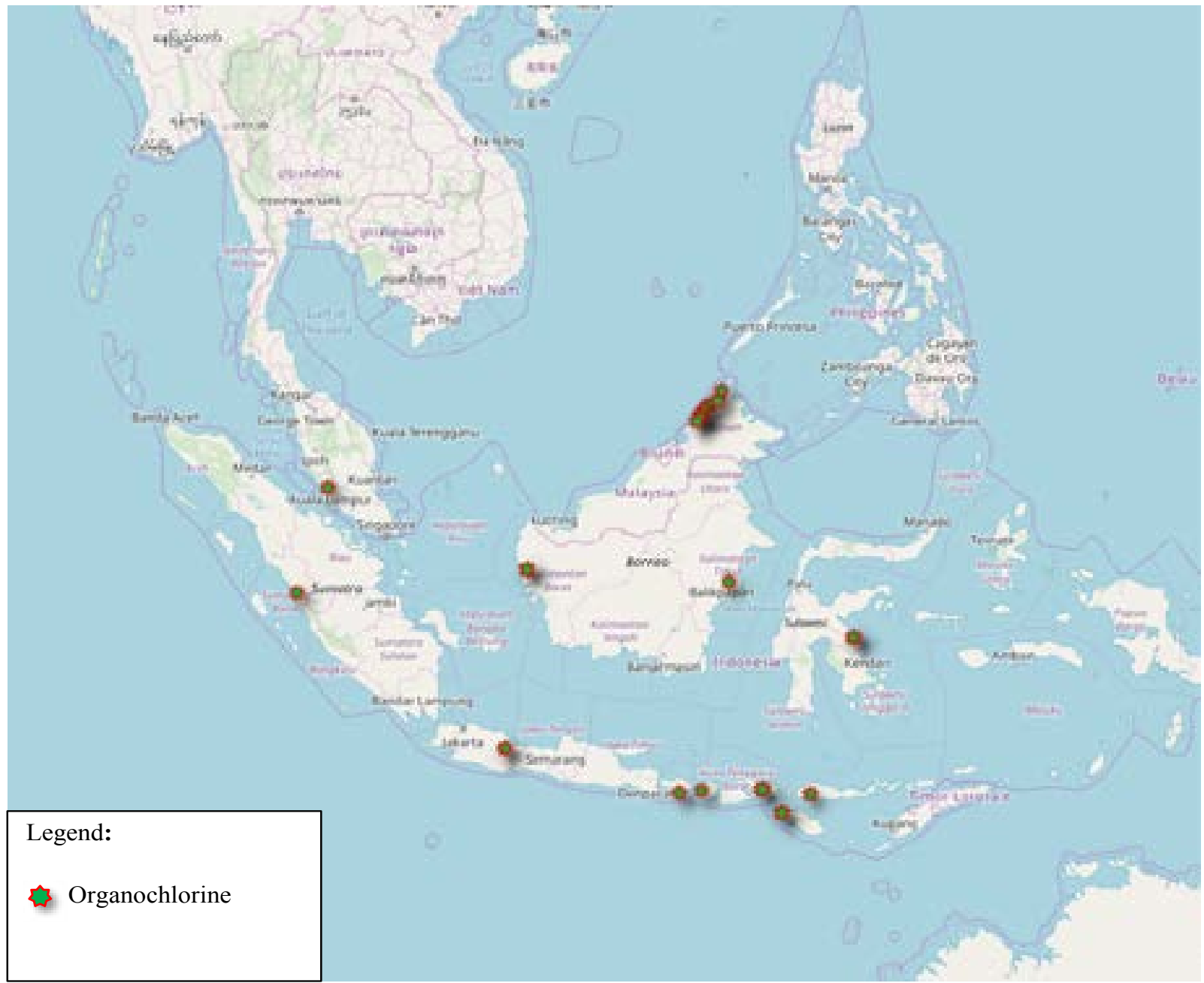

Figure 5 Organochlorine Resistance Based on Published Data from 2015 to 2019

\begin{tabular}{|c|c|c|c|c|c|}
\hline & Author, year & $\begin{array}{l}\text { Location/ } \\
\text { strains }\end{array}$ & Sample & Insecticides & Findings \\
\hline 1 & $\begin{array}{l}\text { (Amelia- } \\
\text { yap, Chen, } \\
\text { Sofian- } \\
\text { Azirun, Lau } \\
\text { et } \\
2018)^{16} \text { al. }\end{array}$ & Indonesia & Ae. aegypti adult & $\begin{array}{l}\text { pyrethroid-based mosquito } \\
\text { coils containing }\end{array}$ & $\begin{array}{l}\text { KT between d-allethrin and transfluthrin, d- } \\
\text { allethrin and metofluthrin, as well } \\
\text { as transfluthrin and metofluthrin displayed } \\
\text { significant associations, portraying the presence } \\
\text { of cross-resistance within pyrethroid } \\
\text { insecticides }\end{array}$ \\
\hline 2 & $\begin{array}{l}\text { (Boyer et al. } \\
2018)^{19}\end{array}$ & Cambodia & $\begin{array}{l}\text { Ae. aegypti adult } \\
\text { and larvae }\end{array}$ & $\begin{array}{l}\text { Temephos }(0.2,0.05,0.03, \\
0.02,0.01,0.004 \mathrm{mg} / \mathrm{L})\end{array}$ & $\begin{array}{l}\text { Larvae- lower mortality rate to temephos. } \\
\begin{array}{l}\text { Adult- highly resistant to permethrin } \\
\text { (pyrethroid) }\end{array}\end{array}$ \\
\hline 3 & $\begin{array}{l}\text { (Chen et al. } \\
\text { 2018) } 20\end{array}$ & Malaysia & Aedes albopictus & Pyrethroid & $\begin{array}{l}\text {-All strains of Ae. albopictus were most } \\
\text { susceptible to metofluthrin, with mortality rates } \\
>80 \% \text {. } \\
\text { Mortality rates ranging from } 5.0 \text { to } 100 \% \text { were } \\
\text { observed from all populations exposed to d- } \\
\text { trans allethrin, d-allethrin, and prallethrin }\end{array}$ \\
\hline 4 & $\begin{array}{l}\text { (Chin et al. } \\
2017)^{21}\end{array}$ & $\begin{array}{l}\text { Malaysia } \\
\text { (11 states) }\end{array}$ & Ae. Aegypti & $\begin{array}{l}\text { metofluthrin } 0.01 \% \quad \mathrm{w} / \mathrm{w} \\
\text { (Fumakilla) } \\
\text { d-allethrin } 0.20 \% \mathrm{w} / \mathrm{w} \text { (Fish } \\
\text { A) } \\
\text { d-trans allethrin } 0.15 \% \mathrm{w} / \mathrm{w} \\
\text { (Shieldtox) } \\
\text { prallethrin } 0.04 \% \\
\text { (Ridsect) }\end{array}$ & $\begin{array}{l}\text {-Significant associations were detected between } \\
\text { the knockdown rates of metofluthrin and d- } \\
\text { allethrin, and between metofluthrin and d-trans } \\
\text { allethrin, suggesting the occurrence of cross- } \\
\text { resistance within pyrethroid insecticides }\end{array}$ \\
\hline
\end{tabular}




\begin{tabular}{|c|c|c|c|c|c|}
\hline 5 & $\begin{array}{l}\text { (Elia-Amira } \\
\text { et al. } \\
2018)^{22}\end{array}$ & $\begin{array}{l}\text { Malaysia, } \\
\text { Sabah }\end{array}$ & Ae. albopictus (Skuse) & $\begin{array}{l}\text { Bromophos, } \\
\text { malathion }(0.125 \mathrm{mg} / \mathrm{L}) \\
\text { fenthion }(0.025 \mathrm{mg} / \mathrm{L}) \\
\text { fenitrothion }(0.02 \mathrm{mg} / \mathrm{L}) \\
\text { temephos }(0.012 \mathrm{mg} / \mathrm{L}) \\
\text { chlorpyrifos }(0.012 \mathrm{mg} / \mathrm{L}) \\
\text { dichlorodiphenyltrichloro- } \\
\text { ethane }(\mathrm{DDT}, 0.012 \mathrm{mg} / \mathrm{L}) \\
\text { dieldrin }(0.050 \mathrm{mg} / \mathrm{L})\end{array}$ & $\begin{array}{l}\text { resistant (mortality }<90 \% \text { ) towards } \\
\text { malathion, temephos, and DDT. } \\
\text { exhibited a wide range of susceptibilities } \\
\text { against bromophos, with mortality ranged from } \\
49.33 \text { to } 93.33 \% \text {. }\end{array}$ \\
\hline 6 & $\begin{array}{l}\text { (P H Hamid } \\
\text { et al. } \\
2018)^{23}\end{array}$ & Indonesia & Ae. aegypti & $\begin{array}{l}5 \% \text { malathion } \\
0.05 \% \text { deltamethrin } \\
0.75 \% \text { permethrin } \\
0.05 \% \text { cyhalothrin } \\
0.1 \% \text { bendiocarb } \\
0.15 \% \text { cyfluthrin }\end{array}$ & $\begin{array}{l}\text { Association in V1016G of Aedes with } \\
\text { resistance to permethrin but not F1534C }\end{array}$ \\
\hline 7 & $\begin{array}{l}\text { (Penny Hum } \\
\text { aidah Hamid } \\
\text {, Prastowo, } \\
\text { Widyasari et } \\
\text { al. 2017) }\end{array}$ & Indonesia & Ae. aegypti & $\begin{array}{l}5 \% \text { malathion } \\
0.05 \% \text { deltamethrin } \\
0.75 \% \text { permethrin } \\
0.05 \% \lambda \text {-cyhalothrin } \\
0.1 \% \text { bendiocarb } \\
0.15 \% \text { cyflothrin }\end{array}$ & $\begin{array}{l}\text { Kdr analysis of voltage-gated sodium channel } \\
\text { (Vgsc) gene showed significant association to } \\
\text { S989P and V1016G mutations linked to } \\
\text { resistance phenotypes against } 0.75 \% \\
\text { permethrin. } \\
\text { Ae. aegypti F1534C gene mutation did not } \\
\text { result in any significant correlation to resistance } \\
\text { development. }\end{array}$ \\
\hline 8 & $\begin{array}{l}\text { (Hasmiwati, } \\
\text { Rusjdi \& No } \\
\text { fita 2018) }\end{array}$ & Indonesia & Ae. aegypti larvae & $\begin{array}{l}\text { Temephos pestanal } 250 \mathrm{mg} \\
97.5 \% \text { with } 0.02 \mathrm{mg} / \mathrm{L}\end{array}$ & $\begin{array}{l}\text { Detection of Ace-1 gene (Genotype variation of } \\
\text { T506T location in Ace-I gene) }\end{array}$ \\
\hline 9 & $\begin{array}{l}\text { (Hasmiwati, } \\
\& \text { Supargiyo } \\
\text { no } 2018)^{26}\end{array}$ & Indonesia & Ae. aegypti larvae & $\begin{array}{l}\text { Temephos postanal } 250 \mathrm{mg} \\
97.5 \% \text {, with } 0.02 \mathrm{mg} / \mathrm{L}\end{array}$ & $\begin{array}{l}\text { populations have point mutations in the VGSC } \\
\text { gene corresponding to S989P and V1016G } \\
\text { amino acid substitutions. } \\
\text { Genes study- to design allele-specific primers to } \\
\text { detect the kdr allele mutations. }\end{array}$ \\
\hline 10 & $\begin{array}{l}\text { (Haziqah- } \\
\text { Rashid et al. } \\
2019)^{27}\end{array}$ & Indonesia & Aedes aegypti larvae & $\begin{array}{l}\text { bromophos (0.050 mg/liter) } \\
\text { chlopyrifos (0.002 mg/liter) } \\
\text { fenitrothion }(0.020 \mathrm{mg} / \text { liter }) \\
\text { fenthion }(0.025 \mathrm{mg} / \text { liter }) \\
\text { malathion }(0.125 \mathrm{mg} / \text { liter }) \\
\text { temephos }(0.012 \mathrm{mg} / \text { liter }) \\
\text { DDT }(0.012 \mathrm{mg} / \text { liter }) \\
\text { dieldrin }(0.025 \mathrm{mg} / \text { liter })\end{array}$ & $\begin{array}{l}\text { All field-collected Ae. aegypti larvae were } \\
\text { resistant against diagnostic doses of } \\
\text { chlorpyrifos, malathion, temephos, and DDT } \\
\text { with mortality rates ranging from } 0 \text { to } 74.67 \% \text {. }\end{array}$ \\
\hline 11 & $\begin{array}{l}\text { (Ishak et al. } \\
2015)^{28}\end{array}$ & Malaysia & $\begin{array}{l}\text { Aedes } \\
\text { aegypti and Aedes } \\
\text { albopictus 2-5-day- } \\
\text { old F2 }\end{array}$ & 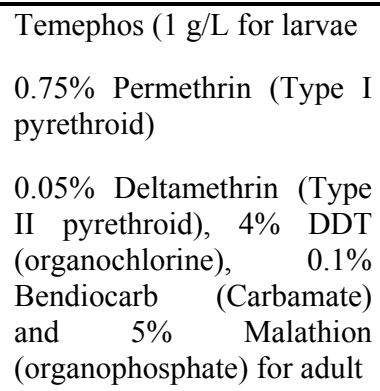 & $\begin{array}{l}\text { Knockdown resistance (kdr) } \\
\text { Synergist assays with PBO (permethrin, } \\
\text { deltamethrin, DDT or bendiocarb) the } 24 \text { hours } \\
\text { mortality is measured }\end{array}$ \\
\hline
\end{tabular}




\begin{tabular}{|c|c|c|c|c|c|}
\hline 12 & $\begin{array}{l}\text { (Ishak et al. } \\
\text { 2017) }^{29}\end{array}$ & Malaysia & Aedes aegypti & $\begin{array}{l}\text { Permethrin } 0.75 \% \\
\text { DDT } 4 \%\end{array}$ & 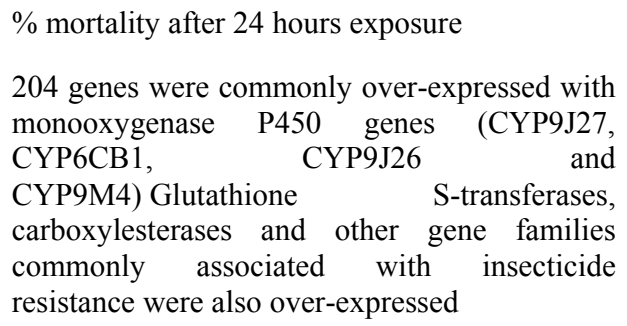 \\
\hline 13 & $\begin{array}{l}\text { (Ishak et al. } \\
2016) 30\end{array}$ & Malaysia & Aedes albopictus & pyrethroid & $\begin{array}{l}\text { Metabolic resistance (cytochrome P450 up- } \\
\text { regulation) and possibly a reduced penetration } \\
\text { mechanism (consistent over-expression of } \\
\text { cuticular protein genes) were associated with } \\
\text { pyrethroid resistance } \\
\text { CYP6P12 over-expression was strongly } \\
\text { associated with pyrethroid resistance } \\
\text { CYP6N3 was over-expressed across carbamate } \\
\text { and DDT resistant populations }\end{array}$ \\
\hline 14 & $\begin{array}{l}\text { (Kasai et al. } \\
2019)^{31}\end{array}$ & $\begin{array}{l}\text { Asia, } \\
\text { Europe } \\
\text { and South } \\
\text { America }\end{array}$ & Aedes albopictus & $\begin{array}{l}\text { permethrin } 5.87 \mathrm{ng} / \text { female, } \\
\text { and } 58.7 \mathrm{ng}\end{array}$ & $\begin{array}{l}\text { Genotyping studies detected a knockdown } \\
\text { resistance }(k d r) \text { allele V1016G in Vssc. F1534C } \\
\text { and F1534S, were also detected }\end{array}$ \\
\hline 15 & $\begin{array}{l}\text { (Kongmee et } \\
\text { al. 2019) }\end{array}$ & Thailand & $\begin{array}{l}\text { Ae. Aegypti, 3-5-day- } \\
\text { old female }\end{array}$ & $\begin{array}{l}\text { deltamethrin } 0.05 \% \\
+/-\quad \text { piperonylbutoxide } \\
\text { (PBO) } 4 \%\end{array}$ & $\begin{array}{l}\text { Mortality after } 24 \text { hours exposure- PBO } \\
\text { increase mortality } \%\end{array}$ \\
\hline 16 & $\begin{array}{l}(\text { Lau et al. } \\
2015)^{33}\end{array}$ & $\begin{array}{l}12 \text { states } \\
\text { in } \\
\text { Malaysia }\end{array}$ & $\begin{array}{l}\text { F1 larvae of } A e . \\
\text { aegypti and Ae. } \\
\text { albopictus }\end{array}$ & $\begin{array}{l}\text { pyriproxyfen } \\
\text { Methoprene } \\
\text { diflubenzuron } \\
\text { 25\%, cyromazine } 75 \%, \\
\text { novaluron } 10 \%,\end{array}$ & $\begin{array}{l}\text { Resistance ratio Insect growth regulators (IGRs; } \\
\text { Insecticide for larva stage: - low resistance was } \\
\text { detected }\end{array}$ \\
\hline 17. & $\begin{array}{l}\text { (Leong et al. } \\
2018)^{34}\end{array}$ & $\begin{array}{l}\text { Selangor, } \\
\text { Malaysia }\end{array}$ & $\begin{array}{l}\text { five - seven days old } \\
\text { adult females Aedes } \\
\text { Aegypti }\end{array}$ & $\begin{array}{l}\text { organochlorine: DDT } \\
(98 \%) \text {; carbamate: } \\
\text { propoxur } \\
(99.8 \%) \text {; organophosphate: } \\
\text { malathion } \\
(98.7 \%) \text {, temephos }(97.5 \%) ; \\
\text { pyrethroids: cyfluthrin } \\
(99.8 \%), \quad \text { deltamethrin } \\
(99.6 \%) \text {, etofenprox } \\
(97.7 \%), \text { lambdacyhalothrin } \\
(97.8 \%) \text { and } \\
\text { permethrin }(98.1 \%)\end{array}$ & $\begin{array}{l}\text { Synergists ethacrynic acid, S.S.S.- } \\
\text { tributylphosphorotrithioate and piperonyl butox } \\
\text { ide increased the toxicity of insecticides but } \\
\text { failed in certain population } \\
\text { Enzyme elevated a-esterase, B - } \\
\text { asterase, glutahion, monoxygenase }\end{array}$ \\
\hline 18 & $\begin{array}{l}\text { (Low et al. } \\
2015)^{35}\end{array}$ & Malaysia & $\begin{array}{l}\text { Aedes } \\
\text { albopictus (Skuse) }\end{array}$ & dieldrin & Presence of the A302S mutation \\
\hline 19 & $\begin{array}{l}\text { (Marcombe } \\
\text { et al. 2018) }\end{array}$ & Lao PDR & $\begin{array}{l}\mathrm{F} 1, \mathrm{~F} 2 \text { larvae of Aedes } \\
\text { aegypti }\end{array}$ & $\begin{array}{l}\text { Bacillus } \\
\text { thuringiensis israelensis (Bti } \\
\text { ), diflubenzuron, } \\
\text { pyriproxyfen } \\
\text { and spinosad diflubenzuron } \\
\text { and temephos }\end{array}$ & $\begin{array}{l}\text { Insecticide susceptibility of Ae. aegypti against } \\
\text { Bacillus thuringiensis israelensis (Bti), } \\
\text { diflubenzuron, pyriproxyfen and spinosad }\end{array}$ \\
\hline
\end{tabular}




\begin{tabular}{|c|c|c|c|c|c|}
\hline 20. & $\begin{array}{l}\text { (Mohiddin et } \\
\text { al. 2016) }\end{array}$ & $\begin{array}{l}\text { Penang, } \\
\text { Malaysia }\end{array}$ & $\begin{array}{l}\text { Aedes } \\
\text { Albopictus larvae }\end{array}$ & $\begin{array}{l}\text { Temephos } 0.012 \text { mg/L } \\
\text { (diagnostic dose), 1mg/L } \\
\text { (operational dose) and } \\
\text { Bacillus } \quad \text { thuringiensis } \\
\text { subsp. israelensis (Bti) } 6000 \\
\text { to 24000 international toxic } \\
\text { unit/L }\end{array}$ & $\begin{array}{l}\text { Higher lethal time and resistance ratio were } \\
\text { detected from Flat Sri Hamna (a dengue hotspot } \\
\text { area }\end{array}$ \\
\hline 21. & $\begin{array}{l}\text { (Mulyanings } \\
\text { ih et al. } \\
2017)^{38}\end{array}$ & Indonesia & Ae. albopictus larvae & organophosphates & $\begin{array}{l}\text { Resistance status of Ae. albopictus larvae } \\
\text { to organophosphates }\end{array}$ \\
\hline 22. & $\begin{array}{l}\text { (Pang et al. } \\
2015)^{39}\end{array}$ & Singapore & Ae. aegypti. & delthamethrin-treated net & $\begin{array}{l}\text { mortality rate } \\
\text { at amino acid residue of alleles V1016G of } \\
\text { DIIS6 or F1269C of DIIIS6 was detected in } \\
93 \% \text { of field strains of Ae. }\end{array}$ \\
\hline 23. & $\begin{array}{l}\text { (Plernsub, et } \\
\text { al. 2016) } 40\end{array}$ & Thailand & Aedes aegypti & deltamethrin & $\begin{array}{l}\text { Susceptibility of knockdown resistance } \\
\text { mutations, S989P, V1016G and F1534C, } \\
\text { in a heterozygous genotype to deltamethrin }\end{array}$ \\
\hline 24. & $\begin{array}{l}\text { (Plernsub, S } \\
\text { aingamsook, } \\
\text { Yanola, Lu } \\
\text { mjuan, Tipp } \\
\text { awangkosol, } \\
\text { Walton, et } \\
\text { al. 2016) }\end{array}$ & $\begin{array}{l}\text { Chiang } \\
\text { Mai city }\end{array}$ & Aedes aegypti & pyrethroids, deltamethrin & $\begin{array}{l}\text { Temporal frequencies of F } 1534 \mathrm{C} \text { and } \\
\text { V1016G in Ae. aegypti populations } \\
\text { The impact of the mutations on the efficacy of } \\
\text { thermal fogging with the pyrethroid } \\
\text { deltamethrin }\end{array}$ \\
\hline 25 & $\begin{array}{l}\text { (Rahim, } \\
2017^{42}\end{array}$ & $\begin{array}{l}\text { Penang, } \\
\text { Malaysia }\end{array}$ & Aedes Alb adult & $\begin{array}{l}\text { Permethrin } \\
\text { Deltamethrin } \\
\text { Malathion } \\
2.4 \% \\
\text { DDT } \\
4 \%\end{array}$ & $\begin{array}{l}\text { This study may assist the health authorities to } \\
\text { improve future chemical-based vector control } \\
\text { operations in dengue-endemic areas }\end{array}$ \\
\hline 26 & $\begin{array}{l}\text { Rahim } \\
2016^{43}\end{array}$ & Malaysia & Aedes Albopictus larva & $\begin{array}{l}\text { Thermophos } \\
\text { Malathion }\end{array}$ & $\begin{array}{l}\text { The revised and established lethal diagnostic } \\
\text { dose findings from the current work are crucial } \\
\text { to elaborate on the variation in susceptibility } \\
\text { of Ae. albopictus in future resistance monitoring } \\
\text { programs in Malaysia. }\end{array}$ \\
\hline
\end{tabular}




\begin{tabular}{|c|c|c|c|c|c|}
\hline 27 & Rasli $2018^{44}$ & Malaysia & Aedes Aegypti & $\begin{array}{l}\text { Permethrin (synthetic } \\
\text { pyrethroid) } \\
\text { deltamethrin and cyfluthrin } \\
\text { lambda-cyhalothrin } \\
\text { malathion }\end{array}$ & $\begin{array}{l}\text { kdr gene and the detoxification of the oxidase } \\
\text { enzyme play a major role in the development of } \\
\text { a pyrethroid resistance in } A \text {. aegypti. } \\
\text { Recommend } \\
\text { Rotational planning of insecticide uses by } \\
\text { substituting pyrethroids with organophosphates } \\
\text { is highly recommended in localities where } A \text {. } \\
\text { aegypti is reportedly highly resistant to } \\
\text { pyrethroids but still susceptible to } \\
\text { organophosphate. } \\
\text { Th- e usage of synergists such } \\
\text { as piperonyl butoxide (PBO) could be } \\
\text { considered in order to overcome the resistance } \\
\text { due to oxidases. } \\
\text { Proactive monitoring of the kdr gene } \\
\text { throughout all dengue-endemic area in } \\
\text { Malaysia is highly suggested as well. }\end{array}$ \\
\hline 28 & $\begin{array}{l}\text { Sayono } 201 \\
6^{45}\end{array}$ & Indonesia & Aedes Aegypti & Pyrethroid & $\begin{array}{l}\text { These findings strongly suggest the need for an } \\
\text { appropriate integrated use of insecticides in the } \\
\text { region. The 989P, 1016G and } 1534 \mathrm{C} \\
\text { polymorphisms in the } A_{a N a_{V} \text { gene are }} \text { ane } \\
\text { potentially valuable molecular markers for } \\
\text { pyrethroid insecticide resistance monitoring. }\end{array}$ \\
\hline 29 & $\begin{array}{l}\text { Smith } \\
2017^{46}\end{array}$ & Singapore & Aedes aegypti & Pyrethroid & $\begin{array}{l}\text { Two mutations S989P + V1016G, commonly } \\
\text { occur together in parts of Asia. These results } \\
\text { provide useful information for resistance } \\
\text { management and for better understanding } \\
\text { pyrethroid interactions with VSSC. }\end{array}$ \\
\hline 30 & $\begin{array}{l}\text { Smith } \\
2018^{47}\end{array}$ & Singapore & Aedes Aegypti & Pyrethrod & $\begin{array}{l}\text { Genetic Cytochrome P450 monooxygenase } \\
\text { (CYP)-mediated detoxification is one of the } \\
\text { primary mechanisms of pyrethroid resistance. }\end{array}$ \\
\hline 31 & $\begin{array}{l}\text { Sun-on P et } \\
\text { al } 2018^{48}\end{array}$ & $\begin{array}{l}\text { Chiang ma } \\
\text { i, Thailand }\end{array}$ & $\begin{array}{l}\text { Kdr strain -Aedes } \\
\text { aegypti } \\
\text { (allele frequency of } \\
\text { S989P+V1016G } \\
\text { mutation) } \\
\text {-DDT, permethrin and } \\
\text { deltamethrin }\end{array}$ & $\begin{array}{l}\text { Pyrethroid } \quad-0.05 \% \\
\text { deltamethrin-impregnated } \\
\text { papers }\end{array}$ & $\begin{array}{l}\text {-effect of relaxation of deltamethrin selection } \\
\text { pressure on the level of mixed-function } \\
\text { oxidases (MFO) } \\
\text {-aids in the development of new control } \\
\text { chemicals, provides information on potential } \\
\text { environmental modulators of resistance, and } \\
\text { allows for the detection of resistance markers } \\
\text { before resistance becomes fixed in the } \\
\text { population } \\
\text { This study indicates that there was a significant } \\
\text { but small reduction in the activity levels of } \\
\text { MFOs when pyrethroid selection pressure is } \\
\text { relaxed in this kdr strain of Ae. aegypti. }\end{array}$ \\
\hline 32 & $\begin{array}{l}\text { Susilowati 2 } \\
018^{49}\end{array}$ & Tangerang & Ae. aegypti & Pyrethroid & $\begin{array}{l}\text { Conclusion is Ae. aegypti from three districts in } \\
\text { Tangerang city have various resistance levels to } \\
\text { pyrethroid }\end{array}$ \\
\hline 33 & $\begin{array}{l}\text { Tangena } 20 \\
1850\end{array}$ & $\begin{array}{l}\text { Lao, } \\
\text { Vientiane } \\
\text { Capital } \\
\text { and Luang } \\
\text { Prabang }\end{array}$ & Aedes albopictus & $\begin{array}{lr}\text { DDT, } & \text { malathion, } \\
\text { permethrin, } & \text { deltamethrin } \\
\text { and, temephos } & \end{array}$ & $\begin{array}{l}\text { Multiple-insecticide resistance was } \\
\text { found. Aedes albopictus control efforts in } \\
\text { villages need to expand to include secondary } \\
\text { forests and rubber plantations, with larval } \\
\text { source management and limited use of } \\
\text { insecticides. }\end{array}$ \\
\hline
\end{tabular}




\begin{tabular}{|c|c|c|c|c|c|}
\hline 34 & $\begin{array}{l}\text { Thanispong } \\
\mathrm{K} 2015^{51}\end{array}$ & $\begin{array}{l}\text { Thailand } \\
\text { Rayong, } \\
\text { Koh } \\
\text { Chang, } \\
\text { and Pong } \\
\text { Nom Ron }\end{array}$ & Aedes albopictus & $\begin{array}{l}0.026 \% \text { deltamethrin, } \\
1.024 \% \text { permethrin, } 0.570 \% \\
\text { bifenthrin, } 0.237 \% \\
\text { cypermethrin, and } 0.035 \% \\
\alpha \text {-cypermethrin) }\end{array}$ & $\begin{array}{l}\text { Routine assessment of these baseline results } \\
\text { should guide future resistance monitoring to } \\
\text { pyrethroid insecticides in Ae. albopictus in } \\
\text { Thailand. }\end{array}$ \\
\hline 35 & $\begin{array}{l}\text { Thongwat } 2 \\
015^{52}\end{array}$ & $\begin{array}{l}\text { Phitsanulo } \\
\text { k Province } \\
\text {, Thailand }\end{array}$ & Aedes Aegypti & $\begin{array}{l}\text { temephos, permethrin and } \\
\text { deltamethrin }\end{array}$ & LC50 and mortality rate \\
\hline 36 & $\begin{array}{l}\text { Widjanarko } \\
2017^{53}\end{array}$ & $\begin{array}{l}\text { Wonosobo } \\
\text { sub- } \\
\text { district, } \\
\text { Indonesia }\end{array}$ & Aedes $s p$ in & $\begin{array}{l}\text { identified that vectors have } \\
\text { already developed resistant } \\
\text { to organophosphate } \\
\text { insecticide, as many as } 50 \% \\
\text { out of the total sample } \\
\text { tested }\end{array}$ & $\begin{array}{l}\text { It is important to use another type of insecticide } \\
\text { such as pyrethroid. }\end{array}$ \\
\hline 37 & $\begin{array}{l}\text { Wuliandri 2 } \\
015^{54}\end{array}$ & $\begin{array}{l}\text { Yogjakart } \\
\text { a, } \\
\text { Indonesia }\end{array}$ & Aedes Aegypti & Pyrethroid & $\begin{array}{l}\text { - } 1023 \mathrm{G} \text { allele is associated with resistance to } \\
\text { type I and type II pyrethroids } \\
\text {-A resistance advantage conferred to V1023G } \\
\text { homozygotes through addition of the S996P } \\
\text { allele in the homozygous form }\end{array}$ \\
\hline
\end{tabular}

\section{Discussion}

Chemical insecticide use is one of the critical components in the Aedes control activity. An insecticide is used to control Aedes species through space spraying, insecticide-treated materials, larvicide and residual spraying 55. Without proper management, the mosquitoes can develop resistance which can affect the dengue control program. Insecticide resistance had become an essential challenge faced in many dengue-endemic countries, particularly in South East Asia ${ }^{56}$. The World Health Organization (WHO) had recommended the insecticide resistance to be included as a part of integrated entomological surveillance to optimise dengue control activities ${ }^{17}$. Unfortunately, not all resistances information was published by the vector control agencies across the SEA countries. The scarce of published information on the resisance studies causes a comprehensive analysis of the regional threat of insecticide resistance is not possible.

Nevertheless, the reporting trend on insecticide resistance on dengue vectors had enriched since the last 10 years from the last published review by Ranson et al. in $2008^{57}$. Furthermore, the methodology used were more standardized, based on the WHO recommendation for the later publications. Based on the available data, the mapping of the resistance status showed the abundance of resistance issues in most of the study sites. Insecticide resistance is indeed a burden to the control pro- grams because it will increase the time and the cost for vector management, especially during the outbreak 56 . Furthermore, agricultural sectors will also be affected by the unplanned usage of pesticides in dengue control. Subsequently, the accumulative burden of dengue transmission, agricultural lost and environmental risk may impact the socioeconomic impact of a population and the country.

Besides, the published data also showed there is a possibility of cross-resistance across the study sites, particularly in Indonesia and Malaysia. Cross-resistance occurs when the resistance to one insecticide confers resistance to another type of insecticide ${ }^{58}$. This is due to the probability of sharing a similar mode of action between the insecticides, or the vector develops mechanism, which is resistance to several types of insecticides. For instance, the cross-resistance between Pyrethroid and DDT, which highlighted in the previous studies ${ }^{59}$. As the consequences, this situation will limit the choices of effective insecticide for vector control. Therefore, effective entomological surveillance is necessary as one of the strategies for effective vector control ${ }^{55}$.

\section{Pyrethroid Resistance}

The most common pyrethroid used is Metofluthrin, Permethrin, Deltamerin and d-Allethrin. Over time, the unplanned and frequent use of insecticides has led to the development of resistance against insecticides in mosquitoes. Based on this review, different levels of resistance seemed to the numerous populations despite 
there is geographical variation in the level of resistance found. This is might due to the intensive use of the chemical with inadequate insecticide management plan $^{60}$. The widespread of the resistance will reduce vector control efficacy and enhanced disease transmission.

The mechanisms of pyrethroid resistance include the mutations in the voltage-sensitive sodium channel gene (target-site resistance) and metabolic-mediated insecticide resistance. The Voltage-sensitive sodium channel (Vssc) gene comprises four homologous domains, and each of them contains six hydrophobic subunits. Mutations occur in the Vssc gene have a linked to knockdown resistance $(\mathrm{kdr})$ in many insect diseases vectors in selected studies. Based on available data, there are three mutations have been detected in Southeast Asia (Indonesia, Thailand, Singapore) which are S989P, V101G and F1534C in Aedes aegypti mosquitoes. The detection of these mutations was affirmed to confer sodium channel resistance to pyrethroids, and other associated mutations are still yet to be inspected because the emergence of new $\mathrm{kdr}$ is possible when pyrethroid insecticides remained to be the first-line defence in the control of Aedes Aegypti.

Metabolic resistance is caused by elevated activity through overexpression or conformational change of enzymes that are involved in the processes of insecticide metabolism, sequestration, and excretion 9 . The metabolic enzymes involved belong to large gene families who are cytochrome P450 monooxygenases (P450), glutathione S-transferases (GST), and carbox$\mathrm{yl} /$ cholinesterase (CCE). Cytochrome P450 monooxygenase (CYP)-mediated detoxification is one of the primary mechanisms of pyrethroid resistance61. Based on available data, only Malaysia and Singapore reported on Cytochrome P450s (P450s) involved in insecticide resistance reduce the efficacy of insecticide-based vector control by rendering vector control ineffective. The CYP9 and CYP6 family play an essential role in insecticide resistance in Aedes Aegyptit ${ }^{28,61}$.

\section{Strategies to Overcome Insecticide Resistance}

The insecticide resistance has become a global issue, and there are few strategies to reduce the development of insecticide resistance. The strategies include the insecticides of unrelated classes with different modes of action should be sprayed in rotation, ideally two times per year (WHO 2016). Rotational planning of insecti- cide uses by substituting pyrethroids with organophosphates is highly recommended in localities where Aedes aegypti is reportedly highly resistant to pyrethroids but still susceptible to organophosphate ${ }^{44}$.

The combination of insecticide with a suitable synergist may help to counter the insecticide resistance and maximise the effectiveness of adulticide operations. The usage of Piperonyl Butoxide (PBO) as synergists could be considered in order to overcome the resistance due to oxidases. For example, the use of Deltamethrin combined with a synergist was effective against resistant populations of Aedes aegypti, the synergistic effect of PBO enhanced knockdown and mortality more rapid by regress the resistance allele and blocked the enzymatic activities that help in detoxification of insecticides 32. Besides that, the indoor thermal fogging of Deltacide containing S-bioallethrin (a knockdown agent) and Deltamethrin (a killing agent) has a strong adulticidal effect, and these formulated mixtures have shown to be effective against both adult and larval Aedes species ${ }^{62}$.

Public health local agencies may also consider a combination of biological and nonbiological strategies in controlling these vectors. The use of natural enemies in biological control such as the larvivorous fish Poecilia reticulata as a food source for mosquito ${ }^{63}$, the entomopathogenic bacteria Bacillus thuringiensis israelensis to destroy the gut lining of mosquito larvae $e^{64,65}$ and the release of Aedes aegypti infected with Wolbachia that cause sterility via cytoplasmic incompatibility ${ }^{64}$. In nonbiological, Petroselinum crispum can be used with other chemicals or measures in integrated mosquito management for controlling the vectors, particularly in localities with high levels of pyrethroid resistance ${ }^{66}$.

Last but not least, in order to delay or prevent the development of insecticide resistance in vector populations, integrated vector management programs should include a resistance management component ${ }^{55}$. Timely, effective entomological assessment and proper data management provided essential information for the management of vector control, and in order to successfully develop and implement of resistance management strategies, knowledge of the mode of action or chemical class of the available insecticide products is crucial ${ }^{17}$.

In Southeast Asia, dengue appears to be endemic. The resistance towards insecticide is geographically varied, 
which is associated with the practice of insecticide usage. Besides, the existence of cross-resistance also shoud be identified, and the role of genetic mutation and mechanism of resistance are among the strategies to optimise vector control management. Among the management strategies are rotation, combination, integration with synergist and routine assessment of resistance profile. Therefore, surveillance on the insecticide resistance should be conducted according to the best practice recommendations. Lastly, the implementation of successful resistance management strategies against both species is urgently needed. Failure of recognition of this issue will lead to poor control of the disease and will escalate the resources needed to control the vector.

\section{Conflict of interest}

None declared.

\section{References}

1. World Health Organization. Comprehensive Guidelines for Prevention and Control of Dengue and Dengue Haemorrhagic Fever.; 2011. http:/ / apps.searo.who. int/pds_docs/B4751.pdf. Accessed June 29, 2019.

2. Bhatia R, Dash A, Sunyoto T. Changing epidemiology of dengue in South-East Asia. WHO South-East Asia J Public Heal. 2013;2(1):23. doi:10.4103/22243151.115830

3. Worrld Health Organization. Dengue and Severe Dengue. doi:10.1371/journal.pntd.0001760

4. Kraemer MUG, Sinka ME, Duda KA, et al. The global distribution of the arbovirus vectors Aedes aegypti and Ae. albopictus. Elife. 2015;4. doi:10.7554/ eLife.08347

5. World Health Organization (Western Pacific Region). Dengue Prevention and Control Program. 2017. https://www.doh.gov.ph/national-dengue-prevention-and-control-program.

6. Wilder-Smith A, Murray MB, Quam M. Epidemiology of dengue: past, present and future prospects. Clin Epidemiol. 2013;5:299. doi:10.2147/CLEP.S34440

7. Ferreira GLC. Global dengue epidemiology trends. Rev Inst Med Trop Sao Paulo. 2012;54(suppl 18):5-6. doi:10.1590/S0036-46652012000700003

8. Liang G, Gao X, Gould EA. Factors responsible for the emergence of arboviruses; strategies, challenges and limitations for their control. Emerg Microbes Infect. 2015;4(3):e18. doi:10.1038/emi.2015.18

9. Moyes CL, Vontas J, Martins AJ, et al. Contemporary status of insecticide resistance in the major Aedes vectors of arboviruses infecting humans. PLoS Negl Trop Dis. 2017;11(7). doi:10.1371/journal.pntd.0005625
10. World Health Organization. Vector Control.; 2017.https://apps.who.int/iris/bitstream/handle $/ 10665 / 258546 /$ sea-rc $70-0 . p d f$ ?sequence $=1 \&$ isAllowed $=y$. Accessed June 29, 2019.

11. Achee NL, Gould F, Perkins TA, et al. A Critical Assessment of Vector Control for Dengue Prevention. PLoS Negl Trop Dis. 2015;9(5). doi:10.1371/journal. pntd.0003655

12. Health Organization W. Global Report on Insecticide Resistance in Malaria Vectors: 2010-2016 Global Malaria Programme.; 2017. https://apps.who.int/iris/ bitstream/handle/10665/272533/9789241514057-eng. pdf?ua=1. Accessed June 29, 2019.

13. Al Nazawi, A.M., Aqili, J., Alzahrani, M. et al. Combined target site $(\mathrm{kdr})$ mutations play a primary role in highly pyrethroid resistant phenotypes of Aedes aegypti from Saudi Arabia. Parasites Vectors. 10, 161 (2017). https://doi.org/10.1186/s13071-017-2096-6

14. David J-P, Ismail HM, Chandor-Proust A, Paine MJI. Role of cytochrome P450s in insecticide resistance: impact on the control of mosquito-borne diseases and use of insecticides on Earth. Philos Trans R Soc B Biol Sci. 2013;368(1612):20120429-20120429. doi:10.1098/ rstb.2012.0429

15. Hemingway J, Hawkes NJ, McCarroll L, Ranson $\mathrm{H}$. The molecular basis of insecticide resistance in mosquitoes. Insect Biochem Mol Biol. 2004;34(7):653-665. doi:10.1016/j.ibmb.2004.03.018

16. Amelia-Yap ZH, Chen CD, Sofian-Azirun M, et al. Efficacy of Mosquito Coils: Cross-resistance to Pyrethroids in Aedes aegypti (Diptera: Culicidae) from Indonesia. J Econ Entomol. 2018;111(6):2854-2860. doi:10.1093/jee/toy296

17. WHO. Monitoring and managing insecticide resistance in Aedes mosquito populations. Who. 2016;16(10665):7. www.who.int. Accessed May 30, 2019.

18. Coleman M, Hemingway J, Gleave KA, Wiebe A, Gething PW, Moyes CL. Developing global maps of insecticide resistance risk to improve vector control. Malar J. 2017;16(1):86. doi:10.1186/s12936-017-1733-z 19. Boyer S, Lopes S, Prasetyo D, et al. Resistance of Aedes aegypti (Diptera: Culicidae) Populations to Deltamethrin, Permethrin, and Temephos in Cambodia. Asia-Pacific J Public Heal. 2018;30(2):158-166. doi:10.1177/1010539517753876

20. Amelia-Yap ZH, Chen CD, Sofian-Azirun M, Low VL. Pyrethroid resistance in the dengue vector Aedes aegypti in Southeast Asia: present situation and prospects for management. Parasit Vectors. 2018;11(1):332. doi:10.1186/s13071-018-2899-0 
21. Chin AC, Chen CD, Low VL, et al. Comparative Efficacy of Commercial Mosquito Coils Against Aedes aegypti (Diptera: Culicidae) in Malaysia: A Nationwide Report. J Econ Entomol. 2017;110(5):2247-2251. doi:10.1093/jee/tox183

22. Elia-Amira NMR, Chen CD, Lau KW, et al. Organophosphate and organochlorine resistance in larval stage of aedes albopictus (Diptera: Culicidae) in Sabah, Malaysia. J Econ Entomol. 2018;111(5):2488-2492. doi:10.1093/jee/toy184

23. Hamid PH, Ninditya VI, Prastowo J, Haryanto A, Taubert A, Hermosilla C. Current Status of Aedes aegypti Insecticide Resistance Development from Banjarmasin, Kalimantan, Indonesia. Biomed Res Int. 2018;2018. doi:10.1155/2018/1735358

24. Hamid PH, Prastowo J, Widyasari A, Taubert A, Hermosilla C. Knockdown resistance $(\mathrm{kdr})$ of the voltage-gated sodium channel gene of Aedes aegypti population in Denpasar, Bali, Indonesia. Parasit Vectors. 2017;10. doi:10.1186/s13071-017-2215-4

25. Hasmiwati, Rusjdi SR, Nofita E. Detection of ace-1 gene with insecticides resistance in aedes aegypti populations from DHF-endemic areas in Padang, Indonesia. Biodiversitas. 2018;19(1):31-36. doi:10.13057/biodiv/ d190105

26. Hasmiwati, Supargiyono. Short Communication: Genotyping of $\mathrm{kdr}$ allele in insecticide resistant-aedes aegypti populations from West Sumatra, Indonesia. Biodiversitas. 2018;19(2):502-508. doi:10.13057/biodiv/ d190225

27. Haziqah-Rashid A, Chen CD, Lau KW, et al. Monitoring Insecticide Resistance Profiles of Aedes aegypti (Diptera: Culicidae) in the Sunda Islands of Indonesia Based on Diagnostic Doses of Larvicides. J Med Entomol. 2019;56(2):514-518. doi:10.1093/jme/tjy208

28. Ishak IH, Jaal Z, Ranson H, Wondji CS. Contrasting patterns of insecticide resistance and knockdown resistance $(\mathrm{kdr})$ in the dengue vectors Aedes aegypti and Aedes albopictus from Malaysia. Parasit Vectors. 2015;8. doi:10.1186/s13071-015-0797-2

29. Ishak IH, Kamgang B, Ibrahim SS, Riveron JM, Irving $\mathrm{H}$, Wondji CS. Pyrethroid Resistance in Malaysian Populations of Dengue Vector Aedes aegypti Is Mediated by CYP9 Family of Cytochrome P450 Genes. PLoS Negl Trop Dis. 2017;11(1). doi:10.1371/journal. pntd.0005302

30. Ishak IH, Riveron JM, Ibrahim SS, et al. The Cytochrome P450 gene CYP6P12 confers pyrethroid resistance in $\mathrm{kdr}$-free Malaysian populations of the dengue vector Aedes albopictus. Sci Rep. 2016;6. doi:10.1038/ srep 24707
31. Kasai S, Caputo B, Tsunoda T, et al. First detection of a Vssc allele V1016G conferring a high level of insecticide resistance in Aedes albopictus collected from Europe (Italy) and Asia (Vietnam), 2016: a new emerging threat to controlling arboviral diseases. Euro Surveill. 2019;24(5). doi:10.2807/1560-7917. ES.2019.24.5.1700847

32. Kongmee M, Thanispong K, Sathantriphop S, Sukkanon C, Bangs MJ, Chareonviriyaphap T. Enhanced mortality in deltamethrin-resistant Aedes aegypti in Thailand using a piperonyl butoxide synergist Monthathip. Acta Trop. 2018. doi:10.1016/j.actatropica.2018.09.025

33. Lau KW, Chen CD, Lee HL, Norma-Rashid Y, Sofian-Azirun M. Evaluation of insect growth regulators against field-collected aedes aegypti and aedes albopictus (Diptera: Culicidae) from Malaysia. J Med Entomol. 2015;52(2):199-206. doi:10.1093/jme/tju019

34. Leong CS, Vythilingam I, Wong ML, Wan Sulaiman W-Y, Lau YL. Aedes aegypti(Linnaeus) larvae from dengue outbreak areas in Selangor showing resistance to pyrethroids but susceptible to organophosphates. Acta Trop. 2018;185:115-126. doi:10.1016/j.actatropica.2018.05.008

35. Low VL, Vinnie-Siow WY, Lim YAL, et al. First molecular genotyping of A302S mutation in the gamma aminobutyric acid (GABA) receptor in Aedes albopictus from Malaysia. Trop Biomed. 2015;32(3):554-556. https://www.scopus.com/inward $/$ record.uri? eid $=2$-s2.0-84946909308\&partner$\mathrm{ID}=40 \& \mathrm{md} 5=52 \mathrm{dce} 71 \mathrm{~b} 7477 \mathrm{c} 9426297 \mathrm{c} 863622 \mathrm{dcc} 4 \mathrm{f}$.

36. Marcombe S, Chonephetsarath S, Thammavong P, Brey PT. Alternative insecticides for larval control of the dengue vector Aedes aegypti in Lao PDR: insecticide resistance and semi-field trial study. Parasit Vectors. 2018;11. doi:10.1186/s13071-018-3187-8

37. Mohiddin A, Lasim AM, Zuharah WF. Susceptibility of Aedes albopictus from dengue outbreak areas to temephos and Bacillus thuringiensis subsp. israelensis. Asian Pac J Trop Biomed. 2016;6(4):295-300. doi:10.1016/j.apjtb.2016.01.006

38. Mulyaningsih B, Umniyati SR, Hadianto T. Detection of nonspecific esterase activity in organophosphate resistant strain of aedes albopictus skuse (Diptera: Culicidae) larvae in Yogyakarta, Indonesia. SouthEast Asian J Trop Med Public Health. 2017;48(3):552$560 . \quad$ https://www.scopus.com/inward/record. uri? eid $=2-\mathrm{s} 2.0-85041095456 \&$ partnerID $=40 \& \mathrm{~m}-$ d5=ed421753770893d0ac93df9ca7b0e34b.

39. Pang SC, Chiang LP, Tan CH, Vythilingam I, LamPhua SG, Ng LC. Low efficacy of delthamethrin-treat- 
ed net against singapore aedes aegypti is associated with kdr-type resistance. Trop Biomed. 2015;32(1):140150.https://www.scopus.com/inward/record. uri?eid $=2-\mathrm{s} 2.0-84924282264 \&$ partnerID $=40 \& \mathrm{md} 5=$ de63fb7c7b344ba4d16d9466bc359c5b.

40. Plernsub S, Saingamsook J, Yanola J, et al. Temporal frequency of knockdown resistance mutations, F1534C and V1016G, in Aedes aegypti in Chiang Mai city, Thailand and the impact of the mutations on the efficiency of thermal fogging spray with pyrethroids. Acta Trop. 2016;162:125-132. doi:10.1016/j.actatropica.2016.06.019

41. Plernsub S, Saingamsook J, Yanola J, et al. Additive effect of knockdown resistance mutations, S989P, V1016G and F1534C, in a heterozygous genotype conferring pyrethroid resistance in Aedes aegypti in Thailand. Parasit Vectors. 2016;9. doi:10.1186/s13071-0161713-0

42. Rahim J, Ahmad AH, Ahmad H, Ishak IH, Rus AC, Maimusa HA. Adulticidal Susceptibility Evaluation of Aedes albopictus Using New Diagnostic Doses in Penang Island, Malaysia. J Am Mosq Control Assoc. 2017;33(3):200-208. doi:10.2987/16-6607R.1

43. Rahim J, Ahmad AH, Kassim NFA, et al. Revised discriminating lethal doses for resistance monitoring program on aedes albopictus against temephos and malathion in Penang island, Malaysia. J Am Mosq Control Assoc. 2016;32(3):210-216. doi:10.2987/16-6556.1

44. Rasli R, Lee HL, Wasi Ahmad N, et al. Susceptibility Status and Resistance Mechanisms in Permethrin-Selected, Laboratory Susceptible and Field-Collected Aedes aegypti from Malaysia. Insects. 2018;9(2). doi: $10.3390 /$ insects 9020043

45. Sayono S, Hidayati APN, Fahri S, et al. Distribution of voltage-gated sodium channel (NAV) alleles among the aedes aegypti populations in central Java province and its aociation with resistance to pyrethroid insecticides. PLoS One. 2016;11(3). doi:10.1371/journal. pone. 0150577

46. Smith EA. Evolutionary and Population Genetics of Mosquito Disease Vectors. ProQuest Diss Theses. 2017. https://search.proquest.com/ docview $/ 1976942588$ ? accountid $=27931$.

47. Smith LB, Tyagi R, Kasai S, Scott JG. CYP-mediated permethrin resistance in Aedes aegypti and evidence for trans-regulation. PLoS Negl Trop Dis. 2018;12(11). doi:10.1371/journal.pntd.0006933

48. Son-Un P, Choovattanapakorn N, Saingamsook J, et al. Effect of relaxation of deltamethrin pressure on metabolic resistance in a pyrethroid-resistant Aedes aegypti (Diptera: Culicidae) strain harboring fixed P989P and G1016G kdr alleles. J Med Entomol. 2018;55(4):975981. doi:10.1093/jme/tjy037

49. Susilowati RP, Sari MP, Far IOF. Bioassay test of the insecticide synthetic pyrethroid against Aedes aegypti mosquito resistance in three districts of Tangerang city. In: C. K, R. A, M.A. P, Y.D. J, A. S, eds. Vol 2021. Departement of Biology, Faculty of Medicine, Krida Wacana Christian University, Jakarta, Indonesia: American Institute of Physics Inc.; 2018. doi:10.1063/1.5062811

50. Tangena J-AA, Marcombe S, Thammavong P, et al. Bionomics and insecticide resistance of the arboviral vector Aedes albopictus in northern Lao PDR. PLoS One. 2018;13(10). doi:10.1371/journal.pone.0206387

51. Thanispong K, Sathantriphop S, Malaithong N, Bangs MJ, Chareonviriyaphap T. Establishment of Diagnostic Doses of Five Pyrethroids for Monitoring Physiological Resistance in Aedes Albopictus in Thailand. J Am Mosq Control Assoc. 2015;31(4):346-352. doi:10.2987/moco-31-04-346-352.1

52. Thongwat D, Bunchu N. Susceptibility to temephos, permethrin and deltamethrin of Aedes aegypti (Diptera: Culicidae) from Muang district, Phitsanulok Province, Thailand. Asian Pac J Trop Med. 2015;8(1):1418. doi:10.1016/S1995-7645(14)60180-2

53. Widjanarko B, Martini M, Hestiningsih R. Resistance status of aedes sp strain from high land in Central Java, Indonesia, as an indicator of increasing vector's capacity of dengue hemorrhagic fever. Ann Trop Med Public Heal. 2017;10(1):71-75. doi:10.4103/ATMPH. ATMPH_78_17

54. Rochmijati Wuliandari J, Lee SF, White VL, Tantowijoyo W, Hoffmann AA, Endersby-Harshman NM. Association between Three Mutations, F1565C, V1023G and S996P, in the Voltage-Sensitive Sodium Channel Gene and Knockdown Resistance in Aedes aegypti from Yogyakarta, Indonesia. Insects. 2015;6(3):658-685. doi:10.3390/insects6030658

55. Roiz D, Wilson AL, Scott TW, et al. Integrated Aedes management for the control of Aedes-borne diseases. Horstick O, ed.PLoS NeglTropDis.2018;12(12):e0006845. doi:10.1371/journal.pntd.0006845

56. Amelia-Yap ZH, Chen CD, Sofian-Azirun M, Low VL. Pyrethroid resistance in the dengue vector Aedes aegypti in Southeast Asia: present situation and prospects for management. Parasit Vectors. 2018;11. doi:10.1186/ s13071-018-2899-0

57. Ranson H, Burhani J, Lumjuan N, Black WC. Review Insecticide resistance in dengue vectors. 2010. http://journal.tropika.net. Accessed June 29, 2019.

58. Insecticide Resistance Action Comittee. Resistance. https://www.irac-online.org/about/resistance/. Accessed June 29, 2019. 
59. Flores-Suarez AE, Ponce-Garcia G, Lopez-Monroy $\mathrm{B}$, et al. Current Status of the Insecticide Resistance in Aedes aegypti (Diptera: Culicidae) from Mexico. In: Insecticides Resistance. InTech; 2016. doi:10.5772/61526

60. Kasai S, Komagata O, Itokawa K, et al. Mechanisms of Pyrethroid Resistance in the Dengue Mosquito Vector, Aedes aegypti : Target Site Insensitivity, Penetration, and Metabolism. PLoS Negl Trop Dis. 2014;8(6). doi:10.1371/journal.pntd.0002948

61. Smith B, Tyagi R, Kasai S, Id JGS. CYP-mediated permethrin resistance in Aedes aegypti and evidence for trans -regulation. PLoS Negl Trop Dis. 2018;12(11):1-13. 62. Mani TR, Arunachalam N, Rajendran R, Satyanarayana K, Dash AP. Efficacy of thermal fog application of deltacide, a synergized mixture of pyrethroids, against Aedes aegypti, the vector of dengue. 2005;10(12):1298-1304. doi:10.1111/j.1365-3156.2005.01522.x

63. Moh C, Setha T, Nealon J, et al. Community-based use of the larvivorous fish Poecilia reticulata to control the dengue vector Aedes aegypti in domestic water stor- age containers in rural Cambodia Community-based use of the larvivorous fish Poecilia reticulata to control the dengue vector. J Vector Ecol. 2008;33(1):139-144.

64. Fraser JE, Bruyne JT De, Iturbe-ormaetxe I, et al. Novel Wolbachia -transinfected Aedes aegypti mosquitoes possess diverse fitness and vector competence phenotypes. PLoS Pathog. 2017;13(12):1-19.

65. Setha T, Chantha N, Benjamin S, Socheat D. Bacterial Larvicide, Bacillus thuringiensis israelensis Strain AM 65-52 Water Dispersible Granule Formulation Impacts Both Dengue Vector, Aedes aegypti (L.) Population Density and Disease Transmission in Cambodia. PLoS Negl Trop Dis. 2016;10(9). doi:10.1371/journal. pntd.0004973

66. Intirach J, Junkum A, Lumjuan N, Chaithong U, Jitpakdi A, Riyong D. Antimosquito property of Petroselinum crispum (Umbellifereae ) against the pyrethroid resistant and susceptible strains of Aedes aegypti ( Diptera : Culicidae ). Environ Sci Pollut Res. 2016. doi:10.1007/s11356-016-7651-8 